\title{
Continuous rational maps into spheres
}

\author{
Wojciech Kucharz ${ }^{1}$
}

Received: 25 September 2014 / Accepted: 7 January 2016 / Published online: 11 February 2016

(C) The Author(s) 2016. This article is published with open access at Springerlink.com

\begin{abstract}
Let $X$ be a compact nonsingular real algebraic variety. We prove that if a continuous map from $X$ into the unit $p$-sphere is homotopic to a continuous rational map, then, under certain assumptions, it can be approximated in the compact-open topology by continuous rational maps. As a byproduct, we also obtain some results on approximation of smooth submanifolds by nonsingular subvarieties.
\end{abstract}

Keywords Real algebraic variety · Regular map · Continuous rational map · Approximation · Homotopy

Mathematics Subject Classification $14 \mathrm{P} 05 \cdot 14 \mathrm{P} 25 \cdot 57 \mathrm{R} 99$

\section{Introduction and main results}

Throughout this paper the term real algebraic variety designates a locally ringed space isomorphic to an algebraic subset of $\mathbb{R}^{n}$, for some $n$, endowed with the Zariski topology and the sheaf of real-valued regular functions (such an object is called an affine real algebraic variety in [3]). Nonsingular varieties are assumed to be of pure dimension. The class of real algebraic varieties is identical with the class of quasi-projective real varieties, cf. [3, Proposition 3.2.10,Theorem 3.4.4]. Morphisms of real algebraic varieties are called regular maps. Each real algebraic variety carries also the Euclidean topology, which is induced by the usual metric on $\mathbb{R}$. Unless explicitly stated otherwise, all topological notions relating to real algebraic varieties refer to the Euclidean topology.

The author was partially supported by NCN grant 2011/01/B/ST1/01289.

Wojciech Kucharz

Wojciech.Kucharz@im.uj.edu.pl

1 Institute of Mathematics, Faculty of Mathematics and Computer Science, Jagiellonian University, Łojasiewicza 6, 30-348 Kraków, Poland 
Let $X$ and $Y$ be real algebraic varieties. A map $f: X \rightarrow Y$ is said to be continuous rational if it is continuous and there exists a Zariski open and dense subvariety $U$ of $X$ such that the restriction $\left.f\right|_{U}: U \rightarrow Y$ is a regular map. Let $X(f)$ denote the union of all such $U$. The complement $P(f)=X \backslash X(f)$ of $X(f)$ is called the irregularity locus of $f$. Thus $P(f)$ is the smallest Zariski closed subvariety of $X$ for which the restriction $\left.f\right|_{X \backslash P(f)}: X \backslash P(f) \rightarrow Y$ is a regular map. If $f(P(f)) \neq Y$, we say that $f$ is a nice map. There exist continuous rational maps that are not nice, cf. [18, Example 2.2 (ii)]. Continuous rational maps have only recently become the object of serious investigation, cf. [11,17-20].

The space $\mathcal{C}(X, Y)$ of all continuous maps from $X$ to $Y$ will always be endowed with the compact-open topology. There are the following inclusions

$$
\mathcal{C}(X, Y) \supseteq \mathcal{R}^{0}(X, Y) \supseteq \mathcal{R}_{0}(X, Y) \supseteq \mathcal{R}(X, Y),
$$

where $\mathcal{R}^{0}(X, Y)$ is the set of all continuous rational maps, $\mathcal{R}_{0}(X, Y)$ consists of the nice maps in $\mathcal{R}^{0}(X, Y)$, and $\mathcal{R}(X, Y)$ is the set of regular maps. By definition, a continuous map from $X$ into $Y$ can be approximated by continuous rational maps if it belongs to the closure of $\mathcal{R}^{0}(X, Y)$ in $\mathcal{C}(X, Y)$. Approximation by nice continuous rational maps or regular maps is defined in the analogous way.

Henceforth we assume that the variety $X$ is compact and nonsingular, and concentrate our attention on maps with values in the unit $p$-sphere

$$
\mathbb{S}^{p}=\left\{\left(u_{0}, \ldots, u_{p}\right) \in \mathbb{R}^{p+1} \mid u_{0}^{2}+\cdots+u_{p}^{2}=1\right\} .
$$

Any continuous map $h: X \rightarrow \mathbb{S}^{p}$ has a neighborhood in $\mathcal{C}\left(X, \mathbb{S}^{p}\right)$ consisting entirely of maps homotopic to $h$. The following two natural questions are of interest:

(Q1) If $h$ is homotopic to a regular map, can it be approximated by regular maps?

(Q2) If $h$ is homotopic to a continuous rational map, can it be approximated by continuous rational maps?

If $\operatorname{dim} X<p$, then the answer to either of these questions is "yes" since $\mathbb{R}^{p}$ is biregularly isomorphic to $\mathbb{S}^{p}$ with one point removed.

Assume then that $\operatorname{dim} X \geq p$. The answer to (Q1) is affirmative for $p \in\{1,2,4\}$, cf. [4] or [3]. The answer to (Q2) is affirmative for $\operatorname{dim} X \leq p+1$ or $p \in\{1,2,4\}$, cf. [19]. Nothing is known about $(\mathrm{Q} 1)$ and $(\mathrm{Q} 2)$ in other cases. Actually, the sets $\mathcal{R}\left(X, \mathbb{S}^{1}\right)$ and $\mathcal{R}^{0}\left(X, \mathbb{S}^{1}\right)$ have equal closures in $\mathcal{C}\left(X, \mathbb{S}^{1}\right)$, cf. [18]. Furthermore, the set $\mathcal{R}_{0}\left(X, \mathbb{S}^{p}\right)$ is dense in $\mathcal{C}\left(X, \mathbb{S}^{p}\right)$ if $\operatorname{dim} X=p$, cf. [19]. On the other hand, the closure of $\mathcal{R}\left(\mathbb{S}^{1} \times \mathbb{S}^{1}, \mathbb{S}^{2}\right)$ in $\mathcal{C}\left(\mathbb{S}^{1} \times \mathbb{S}^{1}, \mathbb{S}^{2}\right)$ coincides with the set of all continuous null homotopic maps, and hence is different from $\mathcal{C}\left(\mathbb{S}^{1} \times \mathbb{S}^{1}, \mathbb{S}^{2}\right)$, cf. [5, Theorem 2.4]; [7, Proposition 2.2]. If $\operatorname{dim} X=p+1$, then the closures of $\mathcal{R}_{0}\left(X, \mathbb{S}^{p}\right)$ and $\mathcal{R}^{0}\left(X, \mathbb{S}^{p}\right)$ in $\mathcal{C}\left(X, \mathbb{S}^{p}\right)$ are identical, cf. [19]. No continuous rational map is known that is not homotopic to a nice continuous rational map. Similarly, no continuous rational map is known that cannot be approximated by nice continuous rational maps. In the present paper we obtain new results, related to (Q1) and (Q2), on nice continuous rational maps. All results announced in this section are proved in Sect. 2.

Theorem 1.1 Let $X$ be a compact nonsingular real algebraic variety and let $p$ be an integer satisfying $\operatorname{dim} X+3 \leq 2 p$. For a continuous map $h: X \rightarrow \mathbb{S}^{p}$, the following conditions are equivalent:

(a) $h$ can be approximated by nice continuous rational maps.

(b) $h$ is homotopic to a nice continuous rational map. 
Our next result requires some preparation.

For any $k$-dimensional compact smooth (of class $\mathcal{C}^{\infty}$ ) manifold $K$, let $[K]$ denote its fundamental class in the homology group $H_{k}(K ; \mathbb{Z} / 2)$. If $T$ is a topological space and $K$ is a subspace of $T$, we denote by $[K]_{T}$ the homology class in $H_{k}(T ; \mathbb{Z} / 2)$ represented by $K$, that is, $[K]_{T}=i_{*}([K])$, where $i: K \hookrightarrow T$ is the inclusion map.

Let $X$ be a nonsingular real algebraic variety. We denote by $A_{k}(X)$ the subgroup of $H_{k}(X ; \mathbb{Z} / 2)$ generated by all homology classes of the form $[Z]_{X}$, where $Z$ is a $k$-dimensional nonsingular Zariski locally closed subvariety of $X$ that is compact and orientable as a smooth manifold. Here $Z$ is not assumed to be Zariski closed in $X$.

For any positive integer $p$, let $s_{p}$ be the unique generator of the cohomology group $H^{p}\left(\mathbb{S}^{p} ; \mathbb{Z} / 2\right) \cong \mathbb{Z} / 2$.

Recall that a smooth manifold is said to be spin if it is orientable and its second StiefelWhitney class vanishes.

Theorem 1.2 Let $X$ be a compact nonsingular real algebraic variety of dimension $p+2$, where $p \geq 5$. Assume that $X$ is a spin manifold. For a continuous map $h: X \rightarrow \mathbb{S}^{p}$, the following conditions are equivalent:

(a) $h$ can be approximated by nice continuous rational maps.

(b) $h$ is homotopic to a nice continuous rational map.

(c) The homology class Poincaré dual to the cohomology class $h^{*}\left(s_{p}\right)$ belongs to $A_{2}(X)$.

Denote by

$$
\rho: H_{*}(-; \mathbb{Z}) \rightarrow H_{*}(-; \mathbb{Z} / 2)
$$

the reduction modulo 2 homomorphism.

Corollary 1.3 Let $X$ be a compact nonsingular real algebraic variety of dimension $p+2$, where $p \geq 5$. Assume that $X$ is a spin manifold. Then the following conditions are equivalent:

(a) Each continuous map from $X$ into $\mathbb{S}^{p}$ can be approximated by nice continuous rational maps.

(b) Each continuous map from $X$ into $\mathbb{S}^{p}$ is homotopic to a nice continuous rational map.

(c) $A_{2}(X)=\rho\left(H_{2}(X ; \mathbb{Z})\right)$.

In some cases, condition (c) in Corollary 1.3 can easily be verified.

Example 1.4 Let $X=C_{1} \times \cdots \times C_{p+2}$, where each $C_{i}$ is a compact connected nonsingular real algebraic curve, $1 \leq i \leq p+2$. If $p \geq 5$, then the set $\mathcal{R}_{0}\left(X, \mathbb{S}^{p}\right)$ of nice continuous rational maps is dense in $\mathcal{C}\left(X, \mathbb{S}^{p}\right)$. Indeed, $A_{2}(X)=H_{2}(X ; \mathbb{Z} / 2)$ and hence the assertion follows from Corollary 1.3.

If $X$ is as in Example 1.4, then in general there exist continuous maps from $X$ into $\mathbb{S}^{p}$ that cannot be approximated by regular maps, cf. [7].

According to [19], for any positive integers $n$ and $p$, the set $\mathcal{R}_{0}\left(\mathbb{S}^{n}, \mathbb{S}^{p}\right)$ of nice continuous rational maps is dense in $\mathcal{C}\left(\mathbb{S}^{n}, \mathbb{S}^{p}\right)$. In this paper we obtain other density results. As in $[3,10]$, given a compact nonsingular real algebraic variety $X$, we denote by $H_{k}^{\text {alg }}(X ; \mathbb{Z} / 2)$ the subgroup of $H_{k}(X ; \mathbb{Z} / 2)$ generated by all homology classes represented by $k$-dimensional Zariski closed (possibly singular) subvarieties of $X$. It easily follows that

$$
A_{k}(X) \subseteq H_{k}^{\mathrm{alg}}(X ; \mathbb{Z} / 2) .
$$


If $k \leq d$ and

$$
H_{k}^{\text {alg }}(X ; \mathbb{Z} / 2)=H_{k}(X ; \mathbb{Z} / 2)
$$

then the Künneth formula implies that

$$
H_{k}^{\mathrm{alg}}\left(X \times \mathbb{S}^{d} ; \mathbb{Z} / 2\right)=H_{k}\left(X \times \mathbb{S}^{d} ; \mathbb{Z} / 2\right) .
$$

Conversely, the latter equality implies the former (with no restriction on $k$ and $d$ ) since

$$
\pi_{*}\left(H_{k}\left(X \times \mathbb{S}^{d} ; \mathbb{Z} / 2\right)\right)=H_{k}(X ; \mathbb{Z} / 2),
$$

where $\pi: X \times \mathbb{S}^{d} \rightarrow X$ is the canonical projecion, and $H_{k}^{\text {alg }}(-; \mathbb{Z} / 2)$ is functorial for regular maps between compact real algebraic varieties, cf. [10, 5.12] or [2, p. 53].

Theorem 1.5 Let $X$ be a compact nonsingular real algebraic variety of dimension $n$. Let d and $p$ be positive integers satisfying $n+1 \leq p$ and $n+2 d+1 \leq 2 p$. If

$$
H_{i}^{\mathrm{alg}}(X ; \mathbb{Z} / 2)=H_{i}(X ; \mathbb{Z} / 2)
$$

for every integer $i$ with $0 \leq i \leq n+d-p$, then the set $\mathcal{R}_{0}\left(X \times \mathbb{S}^{d}, \mathbb{S}^{p}\right)$ of nice continuous rational maps is dense in $\mathcal{C}\left(X \times \mathbb{S}^{d}, \mathbb{S}^{p}\right)$.

It is worthwhile to record the following observation.

Example 1.6 Let $d, n$ and $p$ be positive integers satisfying one of the following two conditions:

(i) $n+d \geq 7$ and $p=n+d-2$;

(ii) $n+1 \leq p$ and $n+2 d+1 \leq 2 p$.

In view of Corollary 1.3 and Theorem 1.5 , the set $\mathcal{R}_{0}\left(\mathbb{S}^{n} \times \mathbb{S}^{d}, \mathbb{S}^{p}\right)$ of nice continuous rational maps is dense in $\mathcal{C}\left(\mathbb{S}^{n} \times \mathbb{S}^{d}, \mathbb{S}^{p}\right)$. Furthermore, by [19, Corollary 1.3, Theorem 1.7], $\mathcal{R}_{0}\left(\mathbb{S}^{n} \times \mathbb{S}^{d}, \mathbb{S}^{p}\right)$ is dense in $\mathcal{C}\left(\mathbb{S}^{n} \times \mathbb{S}^{d}, \mathbb{S}^{p}\right)$ if $n+d-p \leq 1$. It would be interesting to decide whether or not this density assertion holds with no restrictions on $d, n$ and $p$.

We have one more density result.

Theorem 1.7 Let $X$ be a compact nonsingular real algebraic variety of dimension $p$, where $p \geq 5$. If

$$
H_{2}^{\mathrm{alg}}(X ; \mathbb{Z} / 2)=H_{2}(X ; \mathbb{Z} / 2)
$$

and $X$ is a spin manifold, then the set $\mathcal{R}_{0}\left(X \times \mathbb{S}^{2}, \mathbb{S}^{p}\right)$ of nice continuous rational maps is dense in $\mathcal{C}\left(X \times \mathbb{S}^{2}, \mathbb{S}^{p}\right)$.

In topology, one often achieves stabilization effects by making use of the suspension. For problems involving continuous rational maps, the following construction can serve as a substitute for the suspension.

For any positive integer $p$, let

$$
\sigma_{p}: \mathbb{S}^{p} \times \mathbb{S}^{1} \rightarrow \mathbb{S}^{p+1}
$$

be a continuous map such that for some nonempty open subset $U_{p}$ of $\mathbb{S}^{p+1}$, the restriction $\sigma_{U_{p}}: \sigma_{p}^{-1}\left(U_{p}\right) \rightarrow U_{p}$ of $\sigma_{p}$ is a smooth diffeomorphism. We denote by $\mathbb{1}$ the identity map of $\mathbb{S}^{1}$. 
Theorem 1.8 Let $X$ be a compact nonsingular real algebraic variety and let $p$ be a positive integer. For any continuous map $h: X \rightarrow \mathbb{S}^{p}$, the following conditions are equivalent:

(a) The map $\sigma_{p} \circ(h \times \mathbb{1}): X \times \mathbb{S}^{1} \rightarrow \mathbb{S}^{p+1}$ can be approximated by nice continuous rational maps.

(b) The map $\sigma_{p} \circ(h \times \mathbb{1}): X \times \mathbb{S}^{1} \rightarrow \mathbb{S}^{p+1}$ is homotopic to a nice continuous rational map.

In Sect. 2 we derive the results stated above from certain results concerning approximation of smooth submanifolds by nonsingular subvarieties. Approximation of smooth submanifolds, being of independent interest, is further investigated in Sect. 3.

\section{Weak algebraic approximation of smooth submanifolds}

For any smooth manifolds (with possibly nonempty boundary) $N$ and $P$, let $\mathcal{C}^{\infty}(N, P)$ denote the space of all smooth maps from $N$ into $P$ endowed with the $\mathcal{C}^{\infty}$ topology, cf. [13]. The source manifold will always be assumed to be compact, and hence the weak $\mathcal{C}^{\infty}$ topology coincides with the strong one.

Let $X$ be a nonsingular real algebraic variety. A compact smooth submanifold $M$ of $X$ is said to admit a weak algebraic approximation in $X$ if each neighborhood of the inclusion map $M \hookrightarrow X$ in the space $\mathcal{C}^{\infty}(M, X)$ contains a smooth embedding $e: M \rightarrow X$ such that $e(M)$ is a nonsingular Zariski locally closed subvariety of $X$. If $e$ can be chosen so that $e(M)$ is a nonsingular Zariski closed subvariety of $X$, then $M$ is said to admit an algebraic approximation in $X$.

Weak algebraic approximation will be essential for the proofs of Theorems 1.1, 1.2, 1.5, 1.7 and 1.8. It is also of independent interest, cf. [1, Theorems A and F]. In order to avoid unnecessary restrictions, we do not assume that the ambient variety $X$ is compact. Our criteria for weak algebraic approximation are presented in Propositions 2.3 and 2.6.

For any real algebraic variety $V$, let $\operatorname{Reg}(V)$ denote its locus of nonsingular points in dimension $\operatorname{dim} V$.

Lemma 2.1 Let $X$ be a nonsingular real algebraic variety and let $M$ be a compact smooth submanifold of $X$. Assume that there exists a Zariski closed subvariety $A$ of $X$ such that $M \cap \operatorname{Reg}(A)=\varnothing$ and

$$
M \cup \operatorname{Reg}(A)=\partial P,
$$

where $P$ is a compact smooth manifold with boundary $\partial P$, embedded in $X$ with trivial normal bundle and satisfying $P \cap A=\operatorname{Reg}(A)$. If $S=A \backslash \operatorname{Reg}(A)$, then $M \subseteq X \backslash S$ and $M$ admits an algebraic approximation in $X \backslash S$. In particular, $M$ admits a weak algebraic approximation in $X$.

Proof According to Hironaka's theorem on resolution of singularities [12] (cf. also [16] for a very readable exposition), we can assume that $X$ is a Zariski open subvariety of a compact nonsingular real algebraic variety $X^{\prime}$. Note that either $A=\varnothing$ or $\operatorname{Reg}(A)$ is a compact smooth submanifold of $X$. If $A^{\prime}$ is a Zariski closure of $A$ in $X^{\prime}$, then $A=A^{\prime} \cap X$ and $\operatorname{Reg}(A)=\operatorname{Reg}\left(A^{\prime}\right)$. Consequently, we can assume without loss of generality that $X$ is compact. Then it follows directly from the proof of Proposition 2.7 in [19] that $M$ admits an algebraic approximation in $X \backslash S$. Hence, $M$ admits a weak algebraic approximation in $X$. 
Lemma 2.2 Let $X$ be a nonsingular real algebraic variety and let $M$ be a compact smooth submanifold of $X$. Assume that there exists a nonsingular Zariski locally closed subvariety $Z$ of $X$ such that $M \cap Z=\varnothing$ and

$$
M \cup Z=\partial Q,
$$

where $Q$ is a compact smooth manifold with boundary $\partial Q$, embedded in $X$ with trivial normal bundle. If $2 \operatorname{dim} M+1 \leq \operatorname{dim} X$, then $M$ admits a weak algebraic approximation in $X$.

Proof Note that $Z$ is a compact smooth submanifold of $X$. Either $Z=\varnothing$ or $\operatorname{dim} Z=\operatorname{dim} M$. If $A$ is the Zariski closure of $Z$ in $X$, then

$$
Z=\operatorname{Reg}(A) .
$$

Furthermore, $S:=A \backslash Z$ is a Zariski closed subvariety of $X$ with $\operatorname{dim} S<\operatorname{dim} M$. In particular, $S$ has a finite stratification into smooth submanifolds of $X$ of dimension at most $\operatorname{dim} S$.

Assume that $2 \operatorname{dim} M+1 \leq \operatorname{dim} X$ and let $f: Q \hookrightarrow X$ be the inclusion map. Since $\operatorname{dim} Q=\operatorname{dim} M+1$, we get

$$
\operatorname{dim} Q+\operatorname{dim} S<\operatorname{dim} X .
$$

In view of the transversality theorem, there exists a smooth map $g: Q \rightarrow X$, arbitrarily close to $f$ in the space $\mathcal{C}^{\infty}(Q, X)$, such that

$$
\left.g\right|_{\operatorname{Reg}(A)}=\left.f\right|_{\operatorname{Reg}(A)} \text { and } g(Q) \cap S=\varnothing .
$$

If $g$ is sufficiently close to $f$, then it is a smooth embedding isotopic to $f$. In particular, $P:=g(Q)$ is a compact smooth manifold with boundary

$$
\partial P=g(M) \cup \operatorname{Reg}(A),
$$

embedded in $X$ with trivial normal bundle. By construction,

$$
g(M) \cap \operatorname{Reg}(A)=\varnothing \text { and } P \cap A=\operatorname{Reg}(A) .
$$

Hence, according to Lemma 2.1, the smooth submanifold $g(M)$ of $X$ admits a weak algebraic approximation in $X$. Consequently, $M$ admits a weak algebraic approximation in $X$.

Proposition 2.3 Let $X$ be a nonsingular real algebraic variety and let $M$ be a compact smooth submanifold of $X$. Assume that there exists a nonsingular Zariski locally closed subvariety $Z$ of $X$ such that

$$
(M \times\{0\}) \cup(Z \times\{1\})=\partial B,
$$

where $B$ is a compact smooth manifold with boundary $\partial B$, embedded in $X \times \mathbb{R}$ with trivial normal bundle. If $2 \operatorname{dim} M+3 \leq \operatorname{dim} X$, then $M$ admits a weak algebraic approximation in $X$.

Proof Note that $Z$ is a compact smooth submanifold of $X$. Either $Z=\varnothing$ or $\operatorname{dim} Z=\operatorname{dim} M$. Assume that $2 \operatorname{dim} M+3 \leq \operatorname{dim} X$. We can find a small smooth isotopy which transforms $M$ onto a smooth submanifold $M^{\prime}$ of $X$ with $M^{\prime} \cap Z=\varnothing$. Thus there exists a smooth diffeotopy $\varphi: X \times \mathbb{R} \rightarrow X$ such that $\varphi_{0}$ is the identity map and $\varphi_{1}(M)=M^{\prime}$. Here, as usual, $\varphi_{t}(x)=\varphi(x, t)$ for $t$ in $\mathbb{R}$ and $x$ in $X$. The map

$$
\Phi: X \times \mathbb{R} \rightarrow X \times \mathbb{R}, \quad \Phi(x, t)=(\varphi(x, 1-t), t)
$$


is a smooth diffeomorphism. If $B^{\prime}=\Phi(B)$, then

$$
\left(M^{\prime} \times\{0\}\right) \cup(Z \times\{1\})=\partial B^{\prime} .
$$

Therefore, replacing $M$ by $M^{\prime}$ and $B$ by $B^{\prime}$, we can assume that

$$
M \cap Z=\varnothing .
$$

Let $f=\left(f_{1}, f_{2}\right): B \hookrightarrow X \times \mathbb{R}$ be the inclusion map. Since $2 \operatorname{dim} B+1 \leq \operatorname{dim} X$, the smooth map $f_{1}: B \rightarrow X$ can be approximated in the space $\mathcal{C}^{\infty}(B, X)$ by a smooth embedding $g_{1}: B \rightarrow X$. Furthermore, $g_{1}$ can be chosen so that $\left.g_{1}\right|_{\partial B}=\left.f_{1}\right|_{\partial B}$. Note that

$$
\begin{array}{ll}
g_{1}(x, 0)=f_{1}(x, 0)=x & \text { for all } x \text { in } M, \\
g_{1}(x, 1)=f_{1}(x, 1)=x & \text { for all } x \text { in } Z .
\end{array}
$$

By construction, $Q:=g_{1}(B)$ is a compact smooth submanifold of $X$ with boundary

$$
\partial Q=M \cup Z .
$$

In view of Lemma 2.2, it suffices to prove that the normal bundle $v$ to $Q$ in $X$ is trivial. This can be done as follows. The smooth embedding

$$
g: B \rightarrow X \times \mathbb{R}, \quad g(x, t)=\left(g_{1}(x, t), 0\right)
$$

is homotopic to $f$. Since $2 \operatorname{dim} Q+2 \leq \operatorname{dim}(X \times \mathbb{R})$, the smooth embeddings $f$ and $g$ are isotopic, cf. [23, Theorem 6] or [13, p. 183, Exercise 10]. Consequently, the normal bundle to $g(B)$ in $X \times \mathbb{R}$ is trivial, the normal bundle to $f(B)=B$ in $X \times \mathbb{R}$ being trivial. Since $g(B)=Q \times\{0\}$, it follows that the normal bundle $v$ is stably trivial. Now,

$$
\operatorname{rank} v=\operatorname{dim} X-\operatorname{dim} Q \geq \operatorname{dim} Q+1,
$$

and hence $v$ is trivial, cf. [15, p. 100].

Proof of Theorem 1.1 It suffices to prove that (b) implies (a). Suppose that (b) holds. We can assume that the map $h$ is smooth. By Sard's theorem, $h$ is transverse to some point $y$ in $\mathbb{S}^{p}$. Then $M:=h^{-1}(y)$ is a compact smooth submanifold of $X$. According to [18, Theorem 2.4], there exists a nonsingular Zariski locally closed subvariety $Z$ of $X$ such that

$$
(M \times\{0\}) \cup(Z \times\{1\})=\partial B,
$$

where $B$ is a compact smooth manifold with boundary $\partial B$, embedded in $X \times \mathbb{R}$ with trivial normal bundle. In view of Proposition 2.3, $M$ admits a weak algebraic approximation in $X$, which in turn implies that $h$ can be approximated by nice continuous rational maps, cf. [19, Theorem 1.2].

The proof of Theorem 1.2 requires more preparation.

Lemma 2.4 Let $N$ be a smooth spin manifold. Let $P$ be a compact orientable smooth submanifold of $N$, with possibly nonempty boundary. Assume that $2 \operatorname{dim} P+1 \leq \operatorname{dim} N$ and $\operatorname{dim} P \leq 3$. Then the normal bundle to $P$ in $N$ is trivial.

Proof For any smooth manifold $M$, let $\tau_{M}$ denote its tangent bundle. The restriction $\left.\tau_{N}\right|_{P}$ is isomorphic to the direct sum $\tau_{P} \oplus v$. Since $P$ is orientable and $\operatorname{dim} P \leq 3$, it follows that $P$ is a spin manifold. Consequently, the $i$ th Stiefel-Whitney class of $v$ is equal to zero for $i=1,2$. Denote by $D$ the double of $P$ and regard $P$ as a submanifold of $D$. If $r: D \rightarrow P$ is the standard retraction, then the $i$ th Stiefel-Whitney class of the pullback vector bundle $r^{*} v$ 
on $D$ is zero for $i=1,2$. This implies that $r^{*} v$ is stably trivial, cf. [6, Lemma 1.2]. Actually, $r^{*} v$ is trivial since $\operatorname{rank} r^{*} v>\operatorname{dim} D$, cf. [15, p. 100]. Hence the vector bundle $v$ is trivial, being isomorphic to $\left.\left(r^{*} v\right)\right|_{P}$.

For any $k$-dimensional compact oriented smooth manifold $K$, let $\llbracket K \rrbracket$ denote its fundamental class in the homology group $H_{k}(K ; \mathbb{Z})$. If $T$ is a topological space and $K$ is a subspace of $T$, we denote by $\llbracket K \rrbracket_{T}$ the homology class in $H_{k}(T ; \mathbb{Z})$ represented by $K$, that is, $\llbracket K \rrbracket_{T}=i_{*}(\llbracket K \rrbracket)$, where $i: K \hookrightarrow T$ is the inclusion map.

Let $X$ be a nonsingular real algebraic variety. We say that a homology class $u$ in $H_{k}(X ; \mathbb{Z})$ is A-distinguished if it is of the form

$$
u=\llbracket Z \rrbracket_{X},
$$

where $Z$ is a $k$-dimensional nonsingular Zariski locally closed subvariety of $X$ that is compact and oriented as a smooth manifold.

Recall that $\rho: H_{*}(-; \mathbb{Z}) \rightarrow H_{*}(-; \mathbb{Z} / 2)$ denotes the reduction modulo 2 homomorphism.

Lemma 2.5 Let $X$ be a nonsingular real algebraic variety of dimension at least 5 . Assume that $X$ is a spin manifold. For a homology class $u$ in $\mathrm{H}_{2}(X ; \mathbb{Z})$, the following conditions are equivalent:

(a) $u$ is A-distinguished.

(b) $\rho(u)$ belongs to $A_{2}(X)$.

Proof We first prove two preliminary facts.

Assertion $A 1$ If $v_{1}$ and $v_{2}$ are A-distinguished homology classes in $H_{2}(X ; \mathbb{Z})$, then their sum $v_{1}+v_{2}$ is A-distinguished too.

By assumption, $v_{i}:=\llbracket Z_{i} \rrbracket_{X}$, where $Z_{i}$ is a 2-dimensional nonsingular Zariski locally closed subvariety of $X$ that is compact and oriented as a smooth manifold, $i=1,2$. Let $A_{i}$ be the Zariski closure of $Z_{i}$ in $X$. Then

$$
Z_{i}=\operatorname{Reg}\left(A_{i}\right)
$$

and $\operatorname{dim} A_{i}=2$. Furthermore, $S_{i}:=A_{i} \backslash Z_{i}$ is a Zariski closed subvariety of $X$ with $\operatorname{dim} S_{i} \leq$ 1. In particular, $A_{i}$ has a finite stratification into smooth submanifolds of $X$ of dimension at most 2. Similarly, $S_{i}$ has a finite stratification into smooth submanifolds of $X$ of dimension at most 1. According to Lemma 2.4, the normal bundle to $Z_{i}$ in $X$ is trivial. In view of the transversality theorem, there exists a 2-dimensional compact smooth submanifold $M_{i}$ of $X$ such that $M_{i} \cap A_{j}=\varnothing$ for $j=1,2$, and

$$
M_{i} \cup \operatorname{Reg}\left(A_{i}\right)=\partial P_{i},
$$

where $P_{i}$ is a compact smooth manifold with boundary $\partial P_{i}$, embedded in $X$ with trivial normal bundle and satisfying $P_{i} \cap A_{i}=\operatorname{Reg}\left(A_{i}\right)$. We can choose the $M_{i}$ so that

$$
M_{1} \cap M_{2}=\varnothing .
$$

By Lemma 2.1, the smooth submanifold $M_{i}$ admits an algebraic approximation in $X \backslash S_{i}$. Thus, there exists a small smooth isotopy transforming $M_{i}$ onto a nonsingular Zariski closed subvariety $Z_{i}^{\prime}$ of $X \backslash S_{i}$ with $Z_{i}^{\prime} \cap A_{j}=\varnothing$ for $j=1,2$. We can assume that

$$
Z_{1}^{\prime} \cap Z_{2}^{\prime}=\varnothing \text {. }
$$


If $A_{i}^{\prime}$ is the Zariski closure of $Z_{i}^{\prime}$ in $X$, then $\operatorname{Reg}\left(A_{i}^{\prime}\right)=Z_{i}^{\prime}$ and $A_{i}^{\prime} \backslash Z_{i}^{\prime} \subseteq S_{i}$. In particular,

$$
\operatorname{Reg}\left(A_{1}^{\prime} \cup A_{2}^{\prime}\right)=Z_{1}^{\prime} \cup Z_{2}^{\prime} \text {. }
$$

Furthermore, $\llbracket Z_{i}^{\prime} \rrbracket_{X}=\llbracket Z_{i} \rrbracket_{X}$ if $Z_{i}^{\prime}$ is suitably oriented. Consequently,

$$
v_{1}+v_{2}=\llbracket Z_{1} \rrbracket_{X}+\llbracket Z_{2} \rrbracket_{X}=\llbracket Z_{1}^{\prime} \cup Z_{2}^{\prime} \rrbracket_{X}
$$

is an A-distinguished homology class in $H_{2}(X ; \mathbb{Z})$, as required.

Assertion A2 For each homology class $v$ in $H_{2}(X ; \mathbb{Z})$, the homology class $2 v$ is of the form $2 v=\llbracket V \rrbracket_{X}$, where $V$ is a nonsingular Zariski closed subvariety of $X$ that is compact and oriented as a smooth manifold. In particular, $2 v$ is A-distinguished.

We have $v=\llbracket M \rrbracket_{X}$, where $M$ is a 2-dimensional compact oriented smooth submanifold of X, cf. [14,22] or [21, p. 294, Theorem 7.37]. By Lemma 2.4, the normal bundle to $M$ in $X$ is trivial. Hence, there exists a 2-dimensional compact smooth submanifold $M^{\prime}$ of $X$ such that $M^{\prime}$ is isotopic to $M, M \cap M^{\prime}=\varnothing$, and

$$
M \cup M^{\prime}=\partial P,
$$

where $P$ is a compact smooth manifold with boundary $\partial P$, embedded in $X$ with trivial normal bundle. If $M^{\prime}$ is suitably oriented, then $\llbracket M^{\prime} \rrbracket_{X}=\llbracket M \rrbracket_{X}$. Furthermore, by Lemma 2.1 (with $Z=\varnothing$ ), the smooth submanifold $M \cup M^{\prime}$ of $X$ admits an algebraic approximation in $X$. In particular, $M \cup M^{\prime}$ is isotopic to a nonsingular Zariski closed subvariety $V$ of $X$. If $V$ is suitably oriented, then

$$
\llbracket V \rrbracket_{X}=\llbracket M \cup M^{\prime} \rrbracket_{X}=2 \llbracket M \rrbracket_{X}=2 v,
$$

which proves Assertion A2.

If condition (b) holds, then $u$ can be expressed as

$$
u=w_{1}+\cdots+w_{r}+2 w,
$$

where $w_{k}$ and $w$ are homology classes in $H_{2}(X ; \mathbb{Z})$, and each $w_{k}$ is A-distinguished, $1 \leq k \leq r$. Thus, in view of Assertions A1 and A2, condition (a) is satisfied. On the other hand, it is obvious that (a) implies (b).

Proposition 2.6 Let $X$ be a nonsingular real algebraic variety of dimension at least 7 and let $M$ be a 2-dimensional compact orientable smooth submanifold of $X$. Assume that $X$ is a spin manifold. If the homology class $[M]_{X}$ belongs to $A_{2}(X)$, then $M$ admits a weak algebraic approximation in $X$.

Proof Endowing $M$ with an orientation, we get $\rho\left(\llbracket M \rrbracket_{X}\right)=[M]_{X}$. Now assume that $[M]_{X}$ belongs to $A_{2}(X)$. According to Lemma 2.5, the homology class $\llbracket M \rrbracket_{X}$ is A-distinguished. Hence

$$
\llbracket M \rrbracket_{X}=\llbracket Z \rrbracket_{X},
$$

where $Z$ is a 2-dimensional nonsingular Zariski locally closed subvariety of $X$ that is compact and oriented as a smooth manifold. Moving $M$ by a small smooth isotopy, we can assume that

$$
M \cap Z=\varnothing .
$$

The inclusion maps $i: M \hookrightarrow X$ and $j: Z \hookrightarrow X$ represent the same class in the second oriented bordism group $\Omega_{2}(X)$ of $X$. Indeed, this claim holds since the canonical, SteenrodThom, homomorphism

$$
\Omega_{2}(X) \rightarrow H_{2}(X ; \mathbb{Z})
$$


is an isomorphism, cf. [22, p. 75, lines 9, 10] or [21, p. 294,Theorem 7.37]. Consequently, there exists a continuous map $F: B \rightarrow X$, where $B$ is a compact orientable smooth manifold with boundary $\partial B=M \cup Z$, while $\left.F\right|_{M}=i$ and $\left.F\right|_{Z}=j$. Since $\operatorname{dim} B=3$ and $\operatorname{dim} X \geq 7$, we can assume that $F$ is a smooth embedding. In particular, $Q:=F(B) \subseteq X$ is a compact orientable smooth submanifold with boundary

$$
\partial Q=M \cup Z .
$$

According to Lemma 2.4, the normal bundle to $Q$ in $X$ is trivial. Hence, Lemma 2.2 implies that $M$ admits a weak algebraic approximation in $X$.

For any $n$-dimensional compact smooth manifold $N$ and any integer $p$, let

$$
D_{N}: H^{p}(N ; \mathbb{Z} / 2) \rightarrow H_{n-p}(N ; \mathbb{Z} / 2)
$$

denote the Poincaré duality isomorphism.

Lemma 2.7 Let $X$ be a compact nonsingular real algebraic variety of dimension $p+k$, where $p \geq 1$ and $k \geq 0$. Let $f: X \rightarrow \mathbb{S}^{p}$ be a nice continuous rational map. Then $D_{X}\left(f^{*}\left(s_{p}\right)\right)=[Z]_{X}$, where $Z$ is a $k$-dimensional compact nonsingular Zariski locally closed subvariety of $X$ with trivial normal bundle. In particular, if $X$ is orientable, then $D_{X}\left(f^{*}\left(s_{p}\right)\right)$ belongs to $A_{k}(X)$.

Proof Since $f(P(f))$ is a proper compact subset of $\mathbb{S}^{p}$, it follows from Sard's theorem that the regular map $\left.f\right|_{X \backslash P(f)}: X \backslash P(f) \rightarrow \mathbb{S}^{p}$ is transverse to some point $y$ in $\mathbb{S}^{p} \backslash f(P(f))$. Hence $Z:=f^{-1}(y)$ is a compact nonsingular Zariski closed subvariety of $X \backslash P(f)$. It is well known that $D_{X}\left(f^{*}\left(s_{p}\right)\right)=[Z]_{X}$, cf. [10, Proposition 2.15]. Obviously, the normal bundle to $Z$ in $X$ is trivial. If $X$ is orientable, then so is $Z$. The proof is complete.

Proof of Theorem 1.2 Obviously, (a) implies (b), while according to Lemma 2.7, (b) implies (c). It remains to prove that (c) implies (a). Assume that (c) is satisfied. We can assume without loss of generality that $h$ is a smooth map. By Sard's theorem, $h$ is transverse to some point $y$ in $\mathbb{S}^{p}$. Then $M:=h^{-1}(y)$ is a 2-dimensional compact orientable smooth submanifold of $X$ satisfying $D_{X}\left(h^{*}\left(s_{p}\right)\right)=[M]_{X}$, cf. [10, Proposition 2.15]. In particular, the homology class $[M]_{X}$ belongs to $A_{2}(X)$. Hence, according to Proposition 2.6, the submanifold $M$ admits a weak algebraic approximation in X, which implies that $h$ can be approximated by nice continuous rational maps, cf. [19, Theorem 1.2]. In other words, (a) holds.

Proof of Corollary 1.3 By [14,22] or [21, p. 294,Theorem 7.37], every homology class in $H_{2}(X ; \mathbb{Z})$ is of the form $\llbracket M \rrbracket_{X}$, where $M$ is a 2-dimensional compact oriented smooth submanifold of $X$. According to Lemma 2.4, the normal bundle to $M$ in $X$ is trivial, which implies that $\llbracket M \rrbracket_{X}=\rho\left(\llbracket M \rrbracket_{X}\right)=D_{X}\left(h^{*}\left(s_{p}\right)\right)$ for some smooth map $h: X \rightarrow \mathbb{S}^{p}$, cf. [22, Théorème II.2]. In view of Lemma 2.7, $D_{X}\left(h^{*}\left(s_{p}\right)\right)$ belongs to $A_{2}(X)$, provided that $h$ is homotopic to a nice continuous rational map. Consequently, (b) implies (c). According to Theorem 1.2, (c) implies (a). Obviously, (a) implies (b).

For any real algebraic variety $X$, let $\mathfrak{N}_{k}(X)$ denote the $k$ th unoriented boridsm group of $X$. A bordism class in $\mathfrak{N}_{k}(X)$ is said to be algebraic if it can be represented by a regular map from a $k$-dimensional compact nonsingular real algebraic variety into $X, \mathrm{cf}$. [1,2].

Lemma 2.8 Let $X$ be a compact nonsingular real algebraic variety and let $k$ be a nonnegative integer. Assume that

$$
H_{i}^{\operatorname{alg}}(X ; \mathbb{Z} / 2)=H_{i}(X ; \mathbb{Z} / 2)
$$


for every integer $i$ such that $0 \leq i \leq k$ and $\mathfrak{N}_{k-i}$ (point) $\neq 0$. Then each bordism class in $\mathfrak{N}_{k}(X)$ is algebraic.

Proof It suffices to repeat the argument used in the proof of Lemma 2.7.1 in [2].

Proposition 2.9 Let $X$ be a compact nonsingular real algebraic variety of dimension $n$. Let $k$ and $d$ be nonnegative integers satisfying $2 k+1 \leq n$ and $k+1 \leq d$. Assume that

$$
H_{i}^{\mathrm{alg}}(X ; \mathbb{Z} / 2)=H_{i}(X ; \mathbb{Z} / 2)
$$

for $0 \leq i \leq k$. Then any $k$-dimensional compact smooth submanifold of $X \times \mathbb{S}^{d}$ is smoothly isotopic to a nonsingular Zariski locally closed subvariety of $X \times \mathbb{S}^{d}$.

Proof Let $M$ be a $k$-dimensional compact smooth submanifold of $X \times \mathbb{S}^{d}$ and let $f=$ $\left(f_{1}, f_{2}\right): M \hookrightarrow X \times \mathbb{S}^{d}$ be the inclusion map. Since $2 k+1 \leq n$, the map $f_{1}: M \rightarrow X$ is homotopic to a smooth embedding $g_{1}: M \rightarrow X$, cf. [13, p. 55, Theorem 2.13]. The assumtpion $k+1 \leq d$ implies that the map $f_{2}: M \rightarrow \mathbb{S}^{d}$ is homotopic to a constant map $g_{2}: M \rightarrow \mathbb{S}^{d}$. By construction, the map $g=\left(g_{1}, g_{2}\right): M \rightarrow X \times \mathbb{S}^{d}$ is a smooth embedding homotopic to $f$. Since $2 k+2 \leq n+d$, the maps $f$ and $g$ are isotopic, cf. [23, Theorem 6] or [13, p. 183, Exercise 11]. Furthermore, $g(M)=N \times\left\{y_{0}\right\}$, where $N=f_{1}(M)$ and $\left\{y_{0}\right\}=g_{2}(M)$. In particular, the smooth submanifolds $M$ and $N \times\left\{y_{0}\right\}$ of $X \times \mathbb{S}^{d}$ are isotopic. By Lemma 2.8, the unoriented bordism class of the inclusion map $N \hookrightarrow X$ is algebraic. Consequently, since $\mathbb{R}^{d}$ is biregularly isomorphic to $\mathbb{S}^{d}$ with one point removed, it follows from [1, Theorem F] that the smooth submanifold $N \times\left\{y_{0}\right\}$ is isotopic to a nonsingular Zariski locally closed subvariety $Z$ of $X \times \mathbb{S}^{d}$. Hence $M$ is isotopic to $Z$, which completes the proof.

Proof of Theorem 1.5 It suffices to prove that each smooth map $h: X \times \mathbb{S}^{d} \rightarrow \mathbb{S}^{p}$ can be approximated in $\mathcal{C}\left(X \times \mathbb{S}^{d}, \mathbb{S}^{p}\right)$ by nice continuous rational maps. By Sard's theorem, $h$ is transverse to some point $y$ in $\mathbb{S}^{p}$. Then $M:=h^{-1}(y)$ is a compact smooth submanifold of $X \times \mathbb{S}^{d}$ with trivial normal bundle. Either $M=\varnothing$ or $\operatorname{dim} M=n+d-p$. By Proposition 2.9, the submanifold $M$ is isotopic to a nonsingular Zariski localy closed subvariety $Z$ of $X \times \mathbb{S}^{d}$. It follows that

$$
(M \times\{0\}) \cup(Z \times\{1\})=\partial B,
$$

where $B$ is a compact smooth manifold with boundary $\partial B$, embedded in $X \times \mathbb{S}^{p} \times \mathbb{R}$ with trivial normal bundle. In view of Proposition 2.3, $M$ admits a weak algebraic approximation in $X \times \mathbb{S}^{d}$, which in turn implies that $h$ can be aproximated by nice continuous rational maps, cf. [19, Theorem 1.2].

Proof of Theorem 1.7 Each homology class in $\rho\left(H_{2}(X ; \mathbb{Z})\right)$ is of the form $[M]_{X}$ for some 2-dimensional compact orientable smooth submanifold $M$ of $X$, cf. [14,22] or [21, p. 294,Theorem 7.37]. By the Künneth formula, the group $\rho\left(H_{2}\left(X \times \mathbb{S}^{2} ; \mathbb{Z} / 2\right)\right)$ is generated by homology classes of the form $\left[\{x\} \times \mathbb{S}^{2}\right]_{X \times \mathbb{S}^{2}}$ and $[M \times\{y\}]_{X \times \mathbb{S}^{2}}$, where $x \in X$ and $y \in \mathbb{S}^{2}$. According to Lemma 2.8, the unoriented bordism class of the inclusion map $M \hookrightarrow X$ is algebraic (note that $\mathfrak{N}_{1}$ (point) $=0$ ). Consequently, since $\mathbb{R}^{2}$ is biregularly isomorphic to $\mathbb{S}^{2}$ with one point removed, it follows from [1, Theorem F] that the smooth submanifold $M \times\{y\}$ of $X \times \mathbb{S}^{2}$ is isotopic to a nonsingular Zariski locally closed subvariety $Z$ of $X \times \mathbb{S}^{2}$. In particular, $[M \times\{y\}]_{X \times \mathbb{S}^{2}}=[Z]_{X \times \mathbb{S}^{2}}$. Hence

$$
\rho\left(H_{2}\left(X \times \mathbb{S}^{2} ; \mathbb{Z}\right)\right)=A_{2}\left(X \times \mathbb{S}^{2}\right),
$$

which in view of Corollary 1.3 completes the proof. 
We conclude this section by proving the last theorem announced in Sect. 1. The proof does not depend on the results developed above.

Proof of Theorem 1.8 Let $U_{p}$ be a nonempty open subset of $\mathbb{S}^{p+1}$ for which the restriction $\sigma_{U_{p}}: \sigma_{p}^{-1}\left(U_{p}\right) \rightarrow U_{p}$ of $\sigma_{p}$ is a smooth diffeomorphism. We can assume that $h$ is a smooth map. By Sard's theorem, the smooth map $h \times \mathbb{1}: X \times \mathbb{S}^{1} \rightarrow \mathbb{S}^{p} \times \mathbb{S}^{1}$ is transverse to some point $\left(y_{0}, v_{0}\right)$ in $\sigma_{p}^{-1}\left(U_{p}\right)$. In particular, $M:=h^{-1}\left(y_{0}\right)$ is a compact smooth submanifold of $X$. If $z_{0}=\sigma_{p}\left(y_{0}, v_{0}\right)$, then

$$
\left(\sigma_{p} \circ(h \times \mathbb{1})\right)^{-1}\left(z_{0}\right)=M \times\left\{v_{0}\right\} \subseteq X \times \mathbb{S}^{1} .
$$

Assume that (b) holds. According to [18, Theorem 2.4], there exists a nonsingular Zariski locally closed subvariety $Z$ of $X \times \mathbb{S}^{1}$ such that

$$
\left(M \times\left\{v_{0}\right\} \times\{0\}\right) \cup(Z \times\{1\})=\partial P,
$$

where $P$ is a compact smooth manifold with boundary $\partial P$, embedded in $X \times \mathbb{S}^{1} \times \mathbb{R}$ with trivial normal bundle. If $F: P \rightarrow X$ is the restriction of the canonical projection from $X \times \mathbb{S}^{1} \times \mathbb{R}$ onto $X$, then $F\left(x, v_{0}, 0\right)=x$ for all $x$ in $M$, and the restriction $\left.F\right|_{Z \times\{1\}}$ is a regular map. Consequently, the unoriented bordism class of the inclusion map $M \hookrightarrow X$ is algebraic, and hence $M \times\{0\}$ admits a weak algebraic approximation in $X \times \mathbb{R}$, cf. [1, Theorem F]. Since $\mathbb{R}$ is biregularly isomorphic to $\mathbb{S}^{1}$ with one point removed, it follows that $M \times\left\{v_{0}\right\}$ admits a weak algebraic approximation in $X \times \mathbb{S}^{1}$. Thus, in view of [19, Theorem 1.2], the continuous map $\sigma_{p} \circ(h \times \mathbb{1})$ can be approximated by nice continuous rational maps. In other words, (b) implies (a). It is obvious that (a) implies (b).

\section{Algebraic approximation of smooth submanifolds}

Let $X$ be a nonsingular real algebraic variety. A hard problem is to find a characterization of these compact smooth submanifolds $M$ of $X$ which admit an algebraic approximation in $X$. A complete solution is known only if $\operatorname{codim}_{X} M=1$ or $(\operatorname{dim} X, \operatorname{dim} M)=(3,1)$, cf. $[3$, Theorem 14.4.11] and [9]. Very little is known in other cases. As demonstrated in [1], the problem of algebraic approximation is more subtle than that of weak algebraic approximation. In this section, by modifying slightly Propositions 2.3 and 2.6, we obtain results on algebraic approximation.

Proposition 3.1 Let $X$ be a nonsingular real algebraic variety and let $M$ be a compact smooth submanifold of $X$. Assume that there exists a nonsingular Zariski closed subvariety $Z$ of $X$ such that

$$
(M \times\{0\}) \cup(Z \times\{1\})=\partial B,
$$

where $B$ is a compact smooth manifold with boundary $\partial B$, embedded in $X \times \mathbb{R}$ with trivial normal bundle. If $2 \operatorname{dim} M+3 \leq \operatorname{dim} X$, then $M$ admits an algebraic approximation in $X$.

Proof Arguing as in the proof of Proposition 2.3, we can assume that $M \cap Z=\varnothing$ and

$$
M \cup Z=\partial Q,
$$

where $Q$ is a compact smooth manifold with boundary $\partial Q$, embedded in $X$ with trivial normal bundle. Hence, according to Lemma 2.1, $M$ admits an algebraic approximation in $X$. 
It is now convenient to introduce some notation. Let $X$ be a nonsingular real algebraic variety. Denote by $B_{k}(X)$ the subgroup of $H_{k}(X ; \mathbb{Z} / 2)$ generated by all homology classes of the form $[Z]_{X}$, where $Z$ is a $k$-dimensional nonsingular Zariski closed subvariety of $X$ that is compact and orientable as a smooth manifold. Obviously,

$$
B_{k}(X) \subseteq A_{k}(X) .
$$

We say that a homology class $u$ in $H_{k}(X ; \mathbb{Z})$ is B-distinguished if it is of the form

$$
u=\llbracket Z \rrbracket X,
$$

where $Z$ is as above and endowed with an orientation.

The following is a counterpart of Lemma 2.5.

Lemma 3.2 Let $X$ be a nonsingular real algebraic variety of dimension at least 5. Assume that $X$ is a spin manifold. For a homology class $u$ in $H_{2}(X ; \mathbb{Z})$, the following conditions are equivalent:

(a) $u$ is B-distinguished.

(b) $\rho(u)$ belongs to $B_{2}(X)$.

Proof We begin with the following two observations.

Assertion BI If $v_{1}$ and $v_{2}$ are B-distinguished homology classes in $H_{2}(X ; \mathbb{Z})$, then their sum $v_{1}+v_{2}$ is B-distinguished too.

Assertion B2 For each homology class $v$ in $H_{2}(X ; \mathbb{Z})$, the homology class $2 v$ is Bdistinguished.

The proof of Assertion B1 is completely analogous (but simpler) to that of Assertion A1, while Assertion B2 is equivalent to Assertion A2 in the proof of Lemma 2.5.

If condition (b) holds, then $u$ can be expressed as

$$
u=w_{1}+\cdots+w_{r}+2 w,
$$

where $w_{k}$ and $w$ are homology classes in $H_{2}(X ; \mathbb{Z})$, and each $w_{k}$ is B-distinguished, $1 \leq k \leq r$. Thus, in view of Assertions $\mathrm{B} 1$ and $\mathrm{B} 2$, condition (a) is satisfied. It is obvious that (a) implies (b).

Theorem 3.3 Let $X$ be a nonsingular real algebraic variety of dimension at least 7 and let $M$ be a 2-dimensional compact orientable smooth submanifold of $X$. Assume that $X$ is a spin manifold. If the homology class $[M]_{X}$ belongs to $B_{2}(X)$, then $M$ admits an algebraic approximation in $X$.

Proof Endowing $M$ with an orientation, we get $\rho\left(\llbracket M \rrbracket_{X}\right)=[M]_{X}$. Now assume that $[M]_{X}$ belongs to $B_{2}(X)$. According to Lemma 3.2, the homology class $\llbracket M \rrbracket_{X}$ is B-distinguished. Hence

$$
\llbracket M \rrbracket_{X}=\llbracket Z \rrbracket_{X},
$$

where $Z$ is a 2-dimensional nonsingular Zariski closed subvariety of $X$ that is compact and oriented as a smooth manifold. Arguing as in the proof of Proposition 2.6, we can assume that $M \cap Z=\varnothing$ and

$$
M \cup Z=\partial Q,
$$

where $Q$ is a compact smooth manifold with boundary $\partial Q$, embedded in $X$ with trivial normal bundle. Hence, according to Lemma 2.1, $M$ admits an algebraic approximation in $X$. 
It is not known whether the assumptions in Theorem 3.3 can be relaxed. They certainly cannot be relaxed too much. Indeed, for any integers $n$ and $k$ satisfying $n-k \geq 2$ and $k \geq 3$, there exist an $n$-dimensional compact nonsingular real algebraic variety $X$ and a $k$-dimensional compact smooth submanifold $M$ of $X$ such that $[M]_{X}=0$ in $H_{k}(X ; \mathbb{Z} / 2)$ and $M$ does not admit an algebraic approximation in $X$, cf. [9, Proposition 1.2].

As a consequence of Theorem 3.3, we obtain the following.

Example 3.4 Let $X=C_{1} \times \cdots \times C_{n}$, where each $C_{i}$ is a compact connected nonsingular real algebraic curve, $1 \leq i \leq n$. If $n \geq 7$, then each 2-dimensional compact orientable smooth submanifold of $X$ admits an algebraic approximation in $X$. Indeed, $B_{2}(X)=H_{2}(X ; \mathbb{Z} / 2)$ and hence the assertion follows from Theorem 3.3.

Open Access This article is distributed under the terms of the Creative Commons Attribution 4.0 International License (http://creativecommons.org/licenses/by/4.0/), which permits unrestricted use, distribution, and reproduction in any medium, provided you give appropriate credit to the original author(s) and the source, provide a link to the Creative Commons license, and indicate if changes were made.

\section{References}

1. Akbulut, S., King, H.: On approximating submanifolds by algebraic sets and a solution to the Nash conjecture. Invent. Math. 107, 87-98 (1992)

2. Akbulut, S., King, H.: Topology of Real Algebraic Sets Math. Sci. Research Institute Publ., vol. 25. Springer, Berlin (1992). Please check and confirm that the inserted publisher location for the reference $[2,3,13,15,21]$

3. Bochnak, J., Coste, M., Roy, M.-F.: Real Algebraic Geometry Ergeb. Math. Grenzgeb. (3), vol. 36. Springer, Berlin (1998)

4. Bochnak, J., Kucharz, W.: Algebraic approximation of mappings into spheres. Mich. Math. J. 34, 119-125 (1987)

5. Bochnak, J., Kucharz, W.: On real algebraic morphisms into even-dimensional spheres. Ann. of Math. (2) 128, 415-433 (1988)

6. Bochnak, J., Kucharz, W.: K-theory of real algebraic surfaces and threefolds. Math. Proc. Camb. Philos. Soc. 106, 471-480 (1989)

7. Bochnak, J., Kucharz, W.: Elliptic curves and real algebraic morphisms. J. Algebr. Geom. 2, 635-666 (1993)

8. Bochnak, J., Kucharz, W.: On Homology Classes Represented by Real Algebraic Varieties, vol. 44. Banach Center Publications, Warsaw (1998)

9. Bochnak, J., Kucharz, W.: On approximation of smooth submanifolds by nonsingular real algebraic subvarieties. Ann. Scient. Éc. Norm. Sup. (4) 36, 685-690 (2003)

10. Borel, A., Haefliger, A.: La classe d'homologie fondamentale d'un espace analityque. Bull. Soc. Math. Fr. 89, 461-513 (1961)

11. Fichou, G., Huisman, J., Mangolte, F., Monnier, J.-Ph.: Fonctions régulues, arXiv:1112.3800v3 [math.AG], to appear in J. Reine Angew. Math

12. Hironaka, H.: Resolution of singularities of an algebraic variety over a field of characteristic zero. Ann. Math. (2) 79, 109-326 (1964)

13. Hirsch, M.W.: Differential Topology. Springer, Berlin (1997)

14. Hopf, H.: Fundamentalgruppe und zweite Bettische Gruppe. Comment. Math. Helv. 14, 257-309 (1942)

15. Husemoller, D.: Fibre Bundles. Springer, Berlin (1975)

16. Kollár, J.: Lectures on Resolution of Singularities, Ann. of Math. Studies, vol. 166. Princeton University Press, Princeton, NJ (2007)

17. Kollár, J., Nowak, K.J.: Continuous rational functions on real and p-adic varieties. Math. Z. 279, 85-97 (2015)

18. Kucharz, W.: Rational maps in real algebraic geometry. Adv. Geom. 9, 517-539 (2009)

19. Kucharz, W.: Approximation by continuous rational maps into spheres. J. Eur. Math. Soc. 16, 1555-1569 (2014) 
20. Kucharz, W.: Continuous rational maps into the unit 2-sphere. Arch. Math. (Basel) 102, 257-261 (2014)

21. Rudyak, Y.B.: On Thom Spectra, Orientability, and Cobordism, Corrected 2nd Printing. Springer, Berlin (2008)

22. Thom, R.: Quelques propriétés globales des variétés différentiables. Comment. Math. Helv. 28, 17-86 (1954)

23. Whitney, H.: Differentiable manifolds. Ann. Math. 37, 645-680 (1936) 